\title{
Location Time of Power Quality Disturbances and Noise Using Morphology Gradient and Skeletonization in 3D
}

\author{
IGNA Dwijaya Saputra ${ }^{1}$, Q. H. Wu ${ }^{1}$, and W. H. Tang ${ }^{1}$
}

\begin{abstract}
In power system, there are some disturbances such as, voltage dip, momentary interruption, voltage swell, or oscillatory transients that may result in mal-function or failure in operation of some devices. Knowing the location where the disturbances occur in the system is an essential part in selecting the appropriate method on improving power quality issues in order to get effective and efficient results. One of methods in locating power quality disturbances is using Mathematical Morphology (MM). In this paper, signals with disturbances were filtered using morphology gradient. Top-hat transform is applied for the filtered signal using flat structuring element. The simulation results show that the location of disturbances can be detected accurately. Skeletonization is used to identify the location time of the noises in the system. By plotting the results in $3 D$, it makes easier to identify the location of disturbances with or without noises from their different color and shape as pattern recognition.
\end{abstract}

Keywords—Morphology filtering, power quality, Skeletonization, Top-hat transform.

\section{INTRODUCTION}

$\mathrm{T}$ There are some disturbances in power system, such as, voltage dip, momentary interruption, voltage swell, or oscillatory transients that may result in malfunction or failure in operation of some devices. Knowing the location where the disturbances occur in the system can get an effective and efficient result in order to apply the appropriate method on improving power quality issues. Some strategy have been applied for detecting and locating PQ disturbances, such as orthogonal wavelet packet transform [1], embedding strategy [2], TT-transform [3], or pattern recognition classifier based on neural network and genetic algorithm [4]. Other method in locating power quality disturbances is using Mathematical Morphology (MM).

Mathematical Morphology (MM) has been implementing in disturbances detection of the location of power quality events in electrical power system [5] or combined it with complex wavelet [6]. This method uses de-noised wavelet and generalized morphology filter for filtering signal, then applying the complex wavelet of Daubechies real wavelet in order to locate the start and end time of the disturbance occurs. Results of this method show capability in detecting and locating disturbances signal. In addition, calculation time can be reduced and the implementation for hardware can be true easily using this method.

MM also had been applied by combined it with Hilbert-Huang transform (HHT) for detecting and analysing power quality disturbances [7]. In this strategy, traditional mathematical morphological filter (Maragos) is improved in order to effectively suppress noises. Then obtaining a set of intrinsic mode function (IMF) components, the de-noising signal is processed by empirical mode decomposition (EMD).

${ }^{1}$ IGNA Dwijaya Saputra, Q. H. Wu, and W. H. Tang are with Department of Electrical Engineering and Electronics, University of Liverpool, Liverpool L69 3GJ, UK. E-mail: I.DwijayaSaputra@liverpool.ac.uk; q.h.wu@liv.ac.uk; W.Tang@liverpool.ac.uk.
After this process, every IMF component is transformed by using Hilbert transform, so the instantaneous amplitude and frequency of the components can be accurately computed.

In this paper, $\mathrm{MM}$ is implemented to find the location time of disturbances. Signals with disturbances were filtered using morphology gradient. Top-hat transform is applied for the filtered signal using flat structuring element. The simulation was carried out by using Matlab for finding the accurate location of disturbances. This is because of the demand on the improvement of power quality and protection of electrical apparatus or electrical equipment. Some electrical devices are operated base on the precise location time of the disturbances in the system such as, accurate early warning and protection device.

Noise in the system will be recognized as pattern recognition by using Skeletonization. The advantage of this method will be shown in the simulation results for some signals that were injected with additive white Gaussian noise (AWGN).

\section{DESCRIPTION OF WORK}

The location time of power disturbances can be detected using some procedures that have been developed.

\section{A. Basic Operation of Mathematical Morphology}

Basic operations of MM are dilation and erosion and combinations between these two.

1) Dilation

The dilation of sets of pixels $A$ (image) and $B$ (structuring element) is defined as [8]:

$$
\begin{aligned}
A \oplus B & =\bigcup_{b \in \breve{B}}(A)_{b} \\
& =\{x \mid x=a+b ; a \in A ; b \in \check{B}\}
\end{aligned}
$$

where: $a=(x, y)$ and $b=(u, v)$

Dilation is also known as Minkowski addition [9]. The effect of dilation is increasing in size of an object. 
2) Erosion

The erosion of $A$ by $B$ is related to Minkowski substraction.

Then the erosion is defined as:

$$
\begin{aligned}
A \ominus B & =\bigcap_{b \in B}(A)_{b} \\
& =\{x \mid(x+b) \in A ; b \in B\}
\end{aligned}
$$

Different from dilation procedure, the effect of erosion is a shrinking of an image pixels.

\section{B. Design of Morphological Filter}

There are two types of filter in combination of morphological opening and closing, OCF (open-close filter) and COF (close-open filter). OCF is used for filtering salt noises in signal with impulse noise while OCF is applied for filtering pepper noises. The mathematical expression for OCF:

OCF $(f)=(f \circ g 1) \bullet g 2$

For close open filter, we can denoted as follows:

$\mathrm{COF}(\mathrm{f})=(\mathrm{f} \bullet \mathrm{g} 1) \circ \mathrm{g} 2$

where:

$g 1=$ structuring element 1

g2 = structuring element 2

Multiscale structure elements are implemented in the open-close sequence algorithm. For preserve more details of the image, the size of $g 1$ should be small. The morphological opening will eliminate detected salt noise. Consequently, the pepper noises will be increased by this erosion process. In order to remove this additional pepper noises, the following closing operation should be given. It is more importance to choose size of $g 2$ appropriately to eliminate lost on image details and it must be larger than $g 1$ [10].

This OCF and COF can be implemented in vibration signal by using adaptive structuring element. This structuring element will move in vibration signal and then detect and smooth the noises out. One example in non-linearity noise like in cone structures vibration signal shows that these filters have good result not only in removing excessive noise in time or frequency domain, but also in reserving all the attributes of signal [11]. Morphology filter can be written as follows:

$y=0.5[$ OCF $(f)+\operatorname{COF}(f)]$

\section{Morphology Gradient}

MM can be used in detecting power quality problems. It has a good capability for detecting not only the location of the fault, but also the duration of them in time domain [12]. One of techniques in detecting of edge of image or signal is a morphology gradient. There are some fundamental of edge detection operators in mathematical morphology based on boundary detection as can be shown in Equation 6 to 8. Internal boundary can be found using dilation operator on edge detection and it is denoted by:

$\beta_{1}=(\mathrm{f} \oplus \mathrm{g})-\mathrm{f}$

Using erosion edge detection operator, we can find external boundary of the signal that is denoted by:

$\beta_{2}=f-(f \ominus g)$
The basic morphological gradient (MG) is defined as arithmetic difference between the dilation and the erosion by the elementary structuring element (SE) of the considered grid. It means that MG is the subtraction of erosion from dilation, as can be denoted by:

$\rho_{B}(f)=\delta_{B}(f)-\varepsilon_{B}(f)$

In order to detect either the internal or the external boundary of an edge can be done by using the half gradient. The half gradient by erosion or internal gradient $\rho^{--}$is defined as difference between original image and the eroded image:

$\rho_{B}^{-}(f)=f-\varepsilon_{B}(f)$

The half gradient by dilation or internal gradient $\rho^{+}$is defined as difference between original image and the eroded image:

$\rho_{B}^{+}(f)=\delta_{B}(f)-f$

Internal and external gradient are complementary operators:

$\rho_{B}=\rho_{B}^{+}+\rho_{B}^{-}$

The thick gradient will happened if the size of the SE is greater than 1 . It gives the maximum variation in a neighborhood of size $n$.

$\rho_{n B}=\delta_{n B}-\varepsilon_{n B}$

Directional Gradients are defined by replacing the isotropic SE with a line segment $L$ in a given direction $\alpha$ :

$\rho_{L \alpha}=\delta_{L \alpha}-\varepsilon_{L \alpha}$

In order to obtain precise result, the directional perpendicular to smallest directional gradient should be considering rather than defining the largest directional gradient [13].

Mathematic formula for Morphology gradient:

$\mathrm{f}_{\mathrm{grad}}=(\mathrm{f} \oplus \mathrm{g})-(\mathrm{f} \ominus \mathrm{g})$

The gradient peaks are located on the edges, but the internal and external gradients are located at each side of the edges. The internal and external gradients should be thinner than the gradient. The values of all gradient will be positive or non-negative when the value of the structuring element $g(0)$ is bigger or equal to zero.

\section{Location Time of Disturbances}

In order to extract the feature of the signal for the location time of disturbances, Top-hat transform is used for the signal after the Morphology gradient process. Top-hat transform can be described as an operation that will be extracted small elements and detailed of the image. Basic of Top-hat transform is an opening and closing operation.

1) Opening operation:

$\mathrm{f} \circ \mathrm{g}=\mathrm{f} \ominus \mathrm{g} \oplus \mathrm{g}$

2) Closing operation:

$\mathrm{f} \bullet \mathrm{g}=\mathrm{f} \oplus \mathrm{g} \ominus \mathrm{g}$ 
There are two type of the top-hat transform as follows:

1) The white top-hat transform

The white top-hat transform is the difference between the input image and its opening by some structuring element, and it can be denoted by:

$T_{w}(f)=f-f \circ g$

2) The black top-hat transform

The black top-hat transform is the difference between the closing and the input image, and it is given by:

$\mathrm{T}_{\mathrm{b}}(\mathrm{f})=\mathrm{f} \bullet \mathrm{g}-\mathrm{f}$

In this paper, the white Top-Hat transform will be used. So, applying Top-Hat transform of morphological gradient (MG) using flat SE for finding the location time of disturbances can be denoted by:

$\mathrm{f}_{\mathrm{HAT}}=\mathrm{f}_{\text {grad }}-\left(\mathrm{f}_{\text {grad }} \circ \mathrm{g}\right)$

where:

$f_{\text {grad }}=$ morphology gradient of filtered signal

Top-Hat transform is used for extracting the features of the signal with disturbances. The crest signal of disturbances will be extracted by opening process.

After all of the processes above, the signal still has some noises. In order to clean the noises up from the signal, the simple threshold is used where all value of the signal that more than 0.1 will be projected to 1 using equation as follows:

$D_{l o c}=\left\{\begin{array}{l}1, f_{H A T}>0.1 \\ 0, f_{H A T} \leq 0.1\end{array}\right.$

Using this equation, all of noise signal value below 0.1 will be removed.

\section{Noise}

Noise or interference is undesirable electrical signals, which distort or interfere with an original signal. Type of noise could be transient (temporary) or constant. Lightning, for example, can cause unpredictable transient noise, while power circuits or harmonic multiples of power frequency close to the data communications cable can cause constant noise. Sources of noise may come from the system itself (internal noise), or from outside source (external noise). Thermal noise is one of example for internal noise that happen because electron movement within the electrical circuits. Examples for external noise are electromagnetic or radio frequency interference, and cross talk due to a small distance between cables.

Effect of the existence of noise in detection power quality events such as, extracting feature of disturbances from the signal with background noise will be more difficult. Some data will be lost because of this noise. In order to identify noise in the system, we can use Skeletonization method. This Skeletonization morphological method can be denoted by formula as follows:

$(\mathrm{f} \ominus \mathrm{kg})-((\mathrm{f} \ominus \mathrm{kg}) \circ \mathrm{g})$

where: $\mathrm{kg}=$ sequence of $k$ erosion.

\section{RESULT AND DISCUSSION}

\section{A. Location Time of Disturbances}

Matlab simulation have been conducted for identify the location time of some disturbances in the system, such as voltage sag, voltage swell and interruption, under noise-free and noisy condition. Different size of SE were used such as, 3 for morphology gradient and 5 for TopHat method while the sampling frequency is $6.4 \mathrm{kHz}$.

The first step for this method is applying morphology filter and morphology gradient for a signal with disturbances. Then by using top-hat method, initial starting and ending PQ event can be detected. The final step is using projection the signal value upper 0.1 to 1 for determining the location of disturbances in the system.

Figure 1 shows the simulation results of noise-free disturbances respectively (a) disturbance signal, (b) morphology gradient, (c) top hat transform, and (d) result of detecting sag location in the system. In this figure, sag disturbances is can be detected using morphology gradient and top-hat transform, and then projected to 1 to ensure the location time of the disturbances as we can see in fig $1 d$.

For sag disturbance, the filtered signal $\left(f_{\text {grad }}\right)$ is plotted in $3 \mathrm{D}$ in spherical coordinates to make it easier in identify visually, and the result can be seen in Figure 2 . In this figure, the starting and the ending of sag disturbance can be seen in blue and green respectively.

The gap between them shows the lower voltages compare to the normal voltages. By plotting the signal to $3 \mathrm{D}$, the location of the disturbances will be able to identify with different color and different shapes.

In this case, the beginning of the disturbance could be in blue color while the ending in green one. The starting and ending point of the disturbances can be identified in their shapes with bigger circle than the other signal.

The same method was applied for voltage swell and voltage interruption as the result can be seen in Figure 3 to 6 respectively. In these figures, location time of the disturbances can be detected accurately both in beginning points and ending points in the system. So, the duration of the power quality disturbances occurring in the system can be measured.

In this simulation, error in detection of various disturbances location can be seen in Table 1 . Ts in the table is sampling period that equals to $1 / f s$. The result shows that average error of this method is the same as $f_{\text {edge }}$ method [5] with value less than half sampling frequency.

\section{B. Location Time of Noise}

Skeletonization can be applied for the signal to recognize availability of the noise. The system without noise can be seen in Figure 7 . In this figure, normal signal is plotted to $3 \mathrm{D}$ and $2 \mathrm{D}$ for evaluating the availability noise in the system.

When the system was injected by various white Gaussian noises with SNR (signal to noise ratio) with its value from -20 to $50 \mathrm{~dB}$, this method has a capability to detect the location of noise as it can be seen in figure 8 to 12. As we can see in figure 9 to 12 for the 3D projection, the noise can be detected as black color along the coloring cylinder. The different visual for these noises can be seen in the $2 \mathrm{D}$ projection. 
In Figure 12, it is difficult to identify the availability of noise in the first signal with SNR value in 50dB. By Skeletonization morphology, the noise can be detected easily as we can see in the second figure. Plotting it to 3D make it easier to identify the noise as a black color in a cylinder.

\section{CONCLUSION}

From the explanation above, we can make conclusion as follow:

a. Morphology Gradient is followed by Top-hat transform on Mathematical Morphology can be implemented in detecting location of disturbances in power system.

b. The simple threshold is applied after Top-hat transform has the same result compare to morphology edge detection in locating power quality disturbances of noise-free signal especially for voltage swell, sag and interruption with average error for this method is just half sampling frequency.

c. Noise location in the system can be detected by using Skeletonization in black color on 3D plotting, and it makes easier to identify until 50dB.

d. By plotting the filtered signal to $3 \mathrm{D}$, the location of the disturbances will be able to identify with different color and different shapes as pattern recognition.

\section{ACKNOWLEDGEMENT}

The first author wishes thank for the financial support that was provided by Directorate General of High Education, Ministry of Education and Culture Republic of Indonesia (DIKTI).

\section{REFERENCES}

[1]. L. Liu and Z. Zeng, "The detection and location of power quality disturbances based on orthogonal wavelet packet transform,” in Electric Utility Deregulation and Restructuring and Power
Technologies, 2008. DRPT 2008. Third International Conference on, pp. 1831-1835, 2008.

[2]. T. Y. Ji, Q. H. Wu, L. Jiang, and W. H. Tang, "Disturbance detection, location and classification in phase space," Generation, Transmission Distribution, IET, vol. 5, no. 2, pp. 257-265, 2011.

[3]. Q. Yinglin and T. Lijun, "Pattern recognition and time location of power quality disturbances using tt-transform," in Intelligent System Design and Engineering Application (ISDEA), 2010 International Conference on, vol. 2, pp. 53-56, 2010.

[4]. L. Gengyin, Z. Ming, and Z. Zhiyuan, "Research on power quality disturbance automatic recognition and location," in Power Engineering Society General Meeting, 2003, IEEE, vol. 2, pp. 691 Vol. 2, 2003.

[5]. H. Runhong, O. Sen, and S. Yili, "A precise location method of power quality disturbances based on morphological edge detection," in Power and Energy Engineering Conference (APPEEC), 2012 Asia-Pacific, pp. 1-5, 2012.

[6]. C. Xue, L. Hui-jin, Z. Quan-ming, Y. Li-ming, and L. Qing-fen, "Power quality disturbances detection and location using mathematical morphology and complex wavelet transformation," in Industrial Electronics and Applications, 2008. ICIEA 2008. 3rd IEEE Conference on, pp. 2263-2268, 2008.

[7]. Y. Huang, Y. Liu, and Z. Hong, "Detection and location of power quality disturbances based on mathematical morphology and Hilberthuang transform," in Electronic Measurement Instruments, 2009. ICEMI'09. 9th International Conference on, pp. 2-319-2324, 2009.

[8]. S. R. S. R. M. Haralick and X. Zhuang, "Image analysis using mathematical morphology," IEEE Trans. Pattern Anal. Machine Intell. PAMI-9, no. 4, pp. 532-550, 1987.

[9]. R. M. Haralick and L. G. Shapiro, Computer and Robot Vision. Addison-Wesley, 1993.

[10]. D. Ze-Feng, Y. Zhou-ping, and X. You-lun, "High probability impulse noise-removing algorithm based on mathematical morphology," Signal Processing Letters, IEEE, vol. 14, no. 1, pp. 31-34, 2007.

[11]. L. Chunzhi and C. Jiajun, "Mathematical morphological filter and its application in removing noises in vibration signal," in Electronic Measurement and Instruments, 2007. ICEMI '07. 8th International Conference on, pp. 4-101-4-104, 2007.

[12]. Z. Lu, D. R. Turner, Q. H. Wu, J. Fitch, and S. Mann, "Morphological transform for detection of power quality disturbances," in Power System Technology, 2004. PowerCon 2004. 2004 International Conference on, vol. 2, pp. 1644-1649 Vol.2, 2004.

[13]. P. Soille, Morphological Image Analysis: Principles and Applications. New York: Springer-Verlag, 1999.
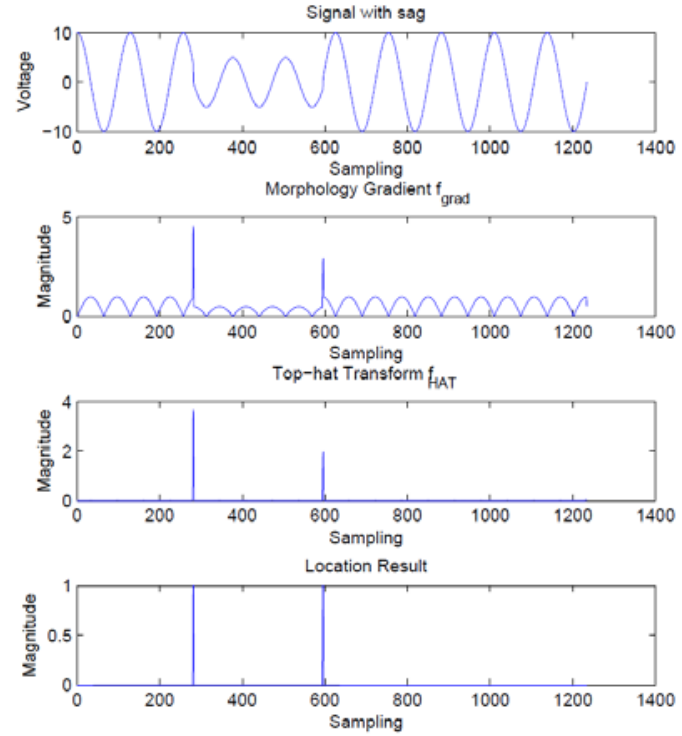

Figure 1. Location time of voltage sag

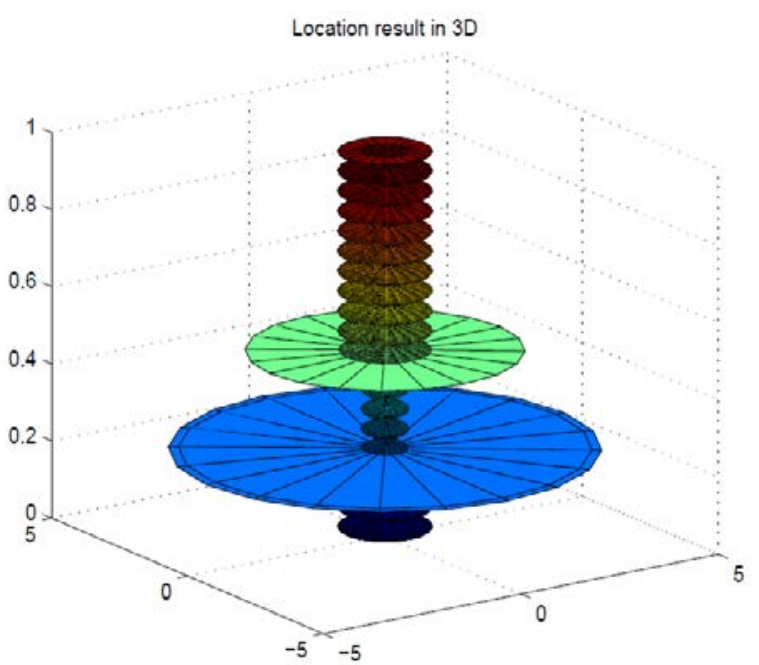

Figure 2. Location time of voltage sag in 3D 

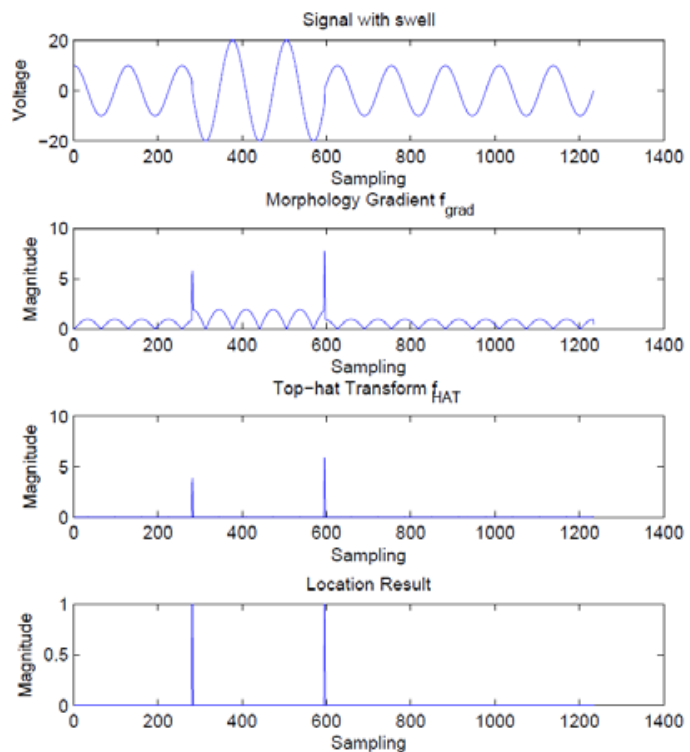

Figure 3. Location time of voltage swell

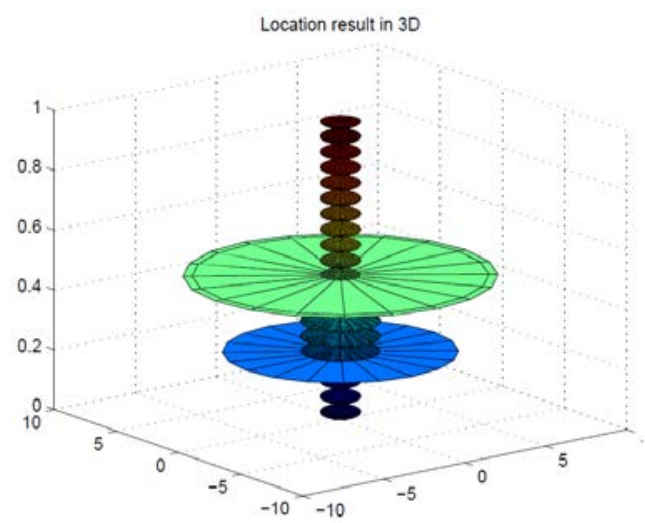

Figure 4. Location time of voltage swell in 3D
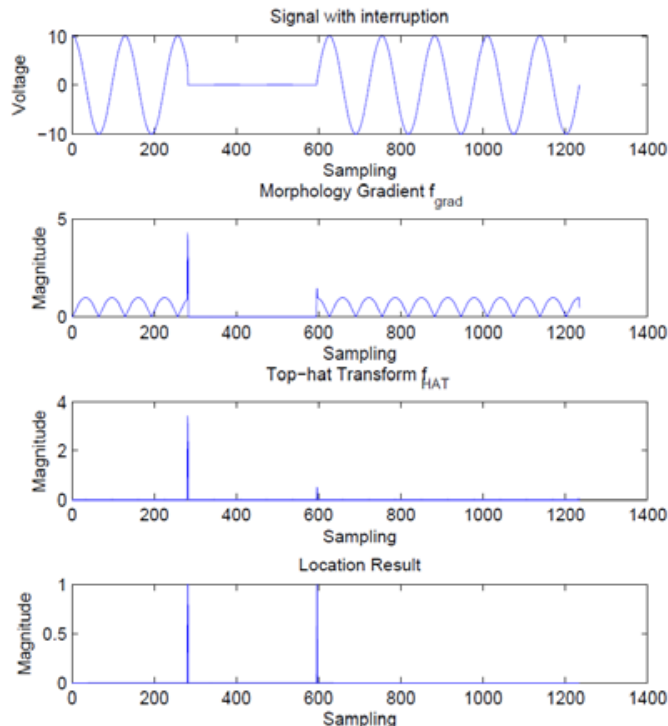

Figure 5. Location time of voltage interruption in 3D

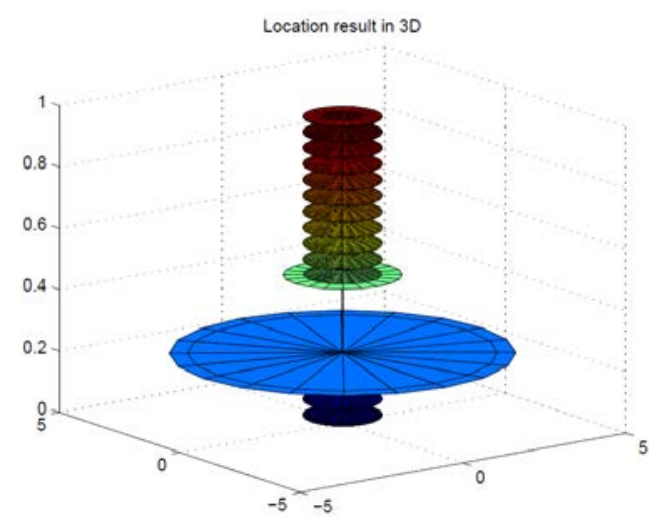

Figure 6. Location time of voltage interruption in 3D
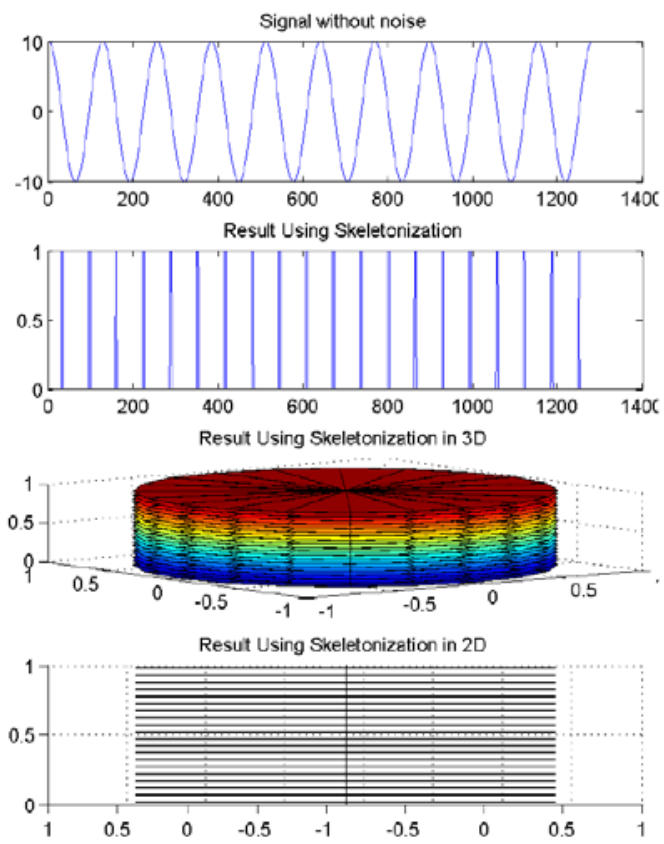

Figure 7. Skeletonization without noise
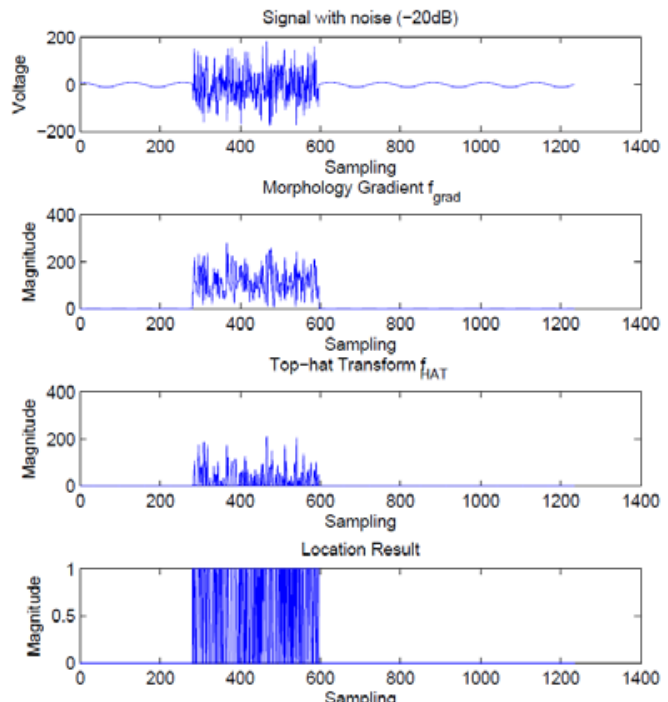

Figure 8. Skeletonization with $\mathrm{SNR}=-20 \mathrm{~dB}$ 

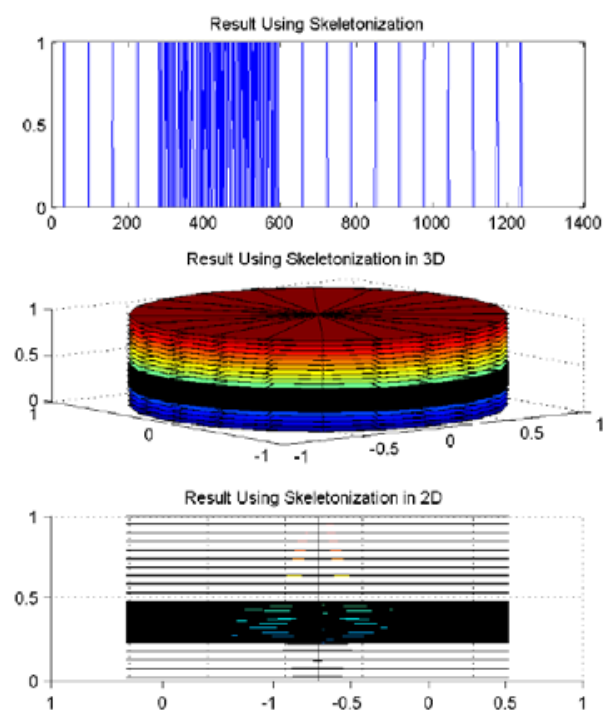

Figure 9. Skeletonization with $\mathrm{SNR}=-20 \mathrm{~dB}$
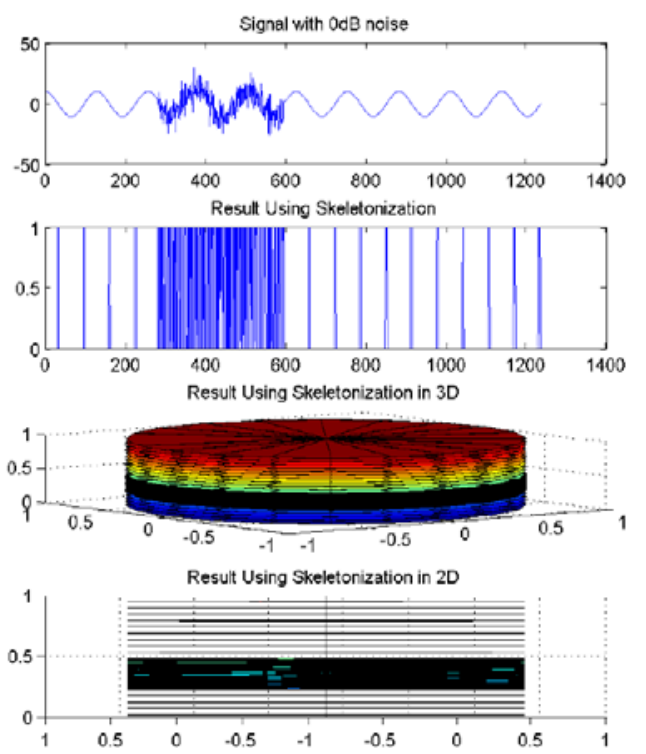

Figure 10. Skeletonization with $\mathrm{SNR}=0 \mathrm{~dB}$
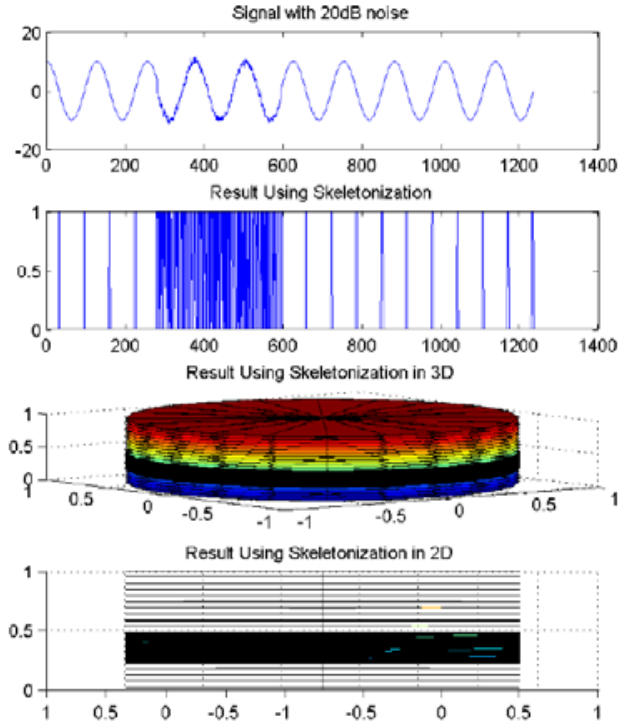

Figure 11. Skeletonization with $\mathrm{SNR}=20 \mathrm{~dB}$
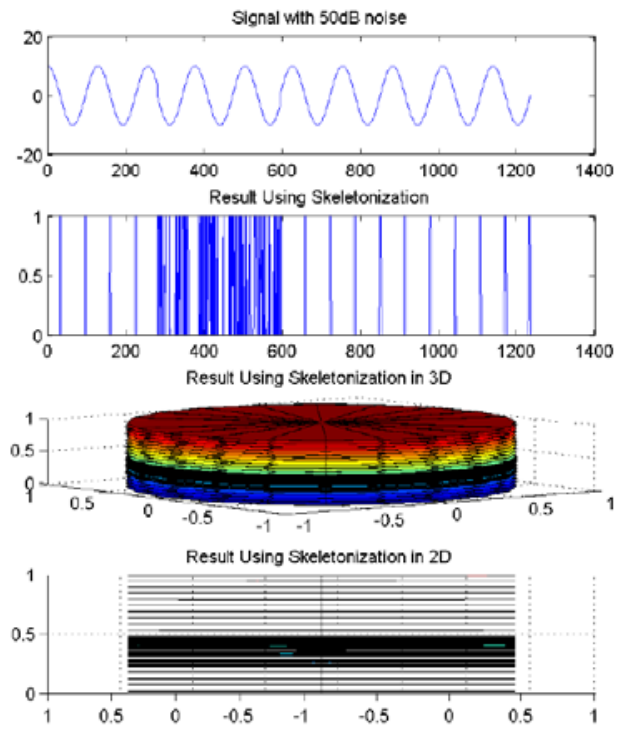

Figure 12. Skeletonization with $\mathrm{SNR}=50 \mathrm{~dB}$

TABLE 1

SIMULATION RESULT

\begin{tabular}{lcccccc}
\hline \hline \multirow{2}{*}{$\begin{array}{l}\text { PQ Events Location } \\
\text { Time }\end{array}$} & \multicolumn{2}{c}{ Voltage Sag } & \multicolumn{2}{c}{ Voltage swell } & \multicolumn{2}{c}{ Voltage Interruption } \\
\cline { 2 - 6 } & begin & end & begin & end & begin & end \\
\hline Actual position (ms) & 44.06 & 93.13 & 44.06 & 93.13 & 44.06 & 93.13 \\
Result (ms) & 43.91 & 93.13 & 44.06 & 92.97 & 43.91 & 93.13 \\
Error/Ts & 1 & 0 & 0 & 1 & 1 & 0 \\
Error/Ts* & 0.5 & 0.5 & 0 & 1 & 0 & 1 \\
\hline \hline
\end{tabular}

Note: * Result using $f_{\text {edge }}$ 